\title{
Le Rabelais ressuscité (1611) de Nicolas de Horry et la tradition des mandements joyeux
}

\section{Rozanne Versendaal ${ }^{1}$}

Published online: 10 December 2019

(c) The Author(s) 2019

\begin{abstract}
This article discusses the role of mandements joyeux or joyful writs in the novel Rabelais ressuscité (1611) by the little-known French author Nicolas de Horry. The article first provides insight into the tradition and parodic nature of joyful writs. In a next step, the joyful writs in Horry's text are identified, and the functions of these parodic passages in the narrative structure of the novel are analysed. Finally, the article demonstrates how an institutional approach to this Early Modern novel, concentrating on the identification of possible readers of the text, can contribute to a better understanding of the critical content of the joyful writs.
\end{abstract}

Keywords Mandement joyeux $\cdot$ Parody $\cdot$ Rabelais ressuscité $\cdot$ Nicolas de Horry University

\section{Introduction}

Le Rabelais ressuscité (1611) de l'écrivain Nicolas de Horry est un petit volume composé de vingt-trois chapitres, qui narre le récit de la vie du géant Grangosier, le père de Gargantua. ${ }^{1}$ Le livre constitue ainsi un «pré-Gargantua», une histoire qui précède celle de Gargantua (Arsenault 2015, 51). Le Rabelais ressuscité raconte la naissance

\footnotetext{
1 Nous avons identifié quatre éditions différentes du Rabelais ressuscité, datant des années 1611, 1614 (deux éditions) et 1615 .

Il existe deux éditions annotées de ce texte, publiées en 1867 par Philomneste Junior, pseudonyme de Gustave Brunet, et en 1976 par Neil Goodley (Brunet 1867; Goodley 1976). Toutes nos citations, ainsi que la numérotation des pages et des lignes, sont issues de l'édition de Goodley. Nous citons cette édi-
}

\footnotetext{
Cet article s'inscrit dans mon projet de thèse. Ma thèse (date prévue: 2020), qui fait partie du projet Uncovering Joyful Culture: Parodic Literature and Practices in and around the Low Countries (13th-17th centuries), porte sur la tradition des mandements joyeux en Europe (XIII - XVII ${ }^{\mathrm{e}}$ siècle), avec un accent spécifique sur les régions francophones et néerlandophones. Ce projet est financé par la NWO, l'Organisation néerlandaise pour la recherche scientifique, et est dirigé par Katell Lavéant (Université d'Utrecht).
}

\section{Rozanne Versendaal} r.m.versendaal@uu.nl

1 Université d'Utrecht (Pays-Bas), Trans 10, 3512 JK Utrecht, The Netherlands 
merveilleuse de Grangosier, son enfance, son éducation, ses études à Paris et ses aventures en tant que roi. Les thèmes principaux évoqués dans le Rabelais ressuscité sont l'ignorance, le vin, la nourriture et la gloutonnerie, ces deux derniers thèmes étant surtout abordés sous l'angle de l'abondance alimentaire pendant diverses festivités (Arsenault 2015, 52). Ces thèmes caractérisent aussi très bien le personnage principal Grangosier: il n'est pas intelligent (il a par exemple fait ses études à «l'université de Peudestudes»), il est vorace («à cause de sa grande voracité [Grangosier avait] tout mangé et devoré les biens et habitans du païs») et ivrogne (Grangosier dit notamment: «j'ay grandement soif, y ayant pres d'un quart d'heure que je n'ay beu, et [...] le breuvage du Dieu Bacchus me manque» et ses camarades de l'université l'appellent «un yvrogne insatiable, qui eust beu deux mil muids de vin pour un repas» (Horry 1611, 9-13)). Même son nom (que l'on pourrait traduire par «[ayant une] grande gorge») confirme cette image d'un personnage gourmand et appréciant la boisson. ${ }^{2}$

On ne sait pratiquement rien de l'auteur de cet ouvrage, Nicolas de Horry. Le Rabelais ressuscité fut d'abord publié sous le pseudonyme de Thibaut le Nattier, un personnage qui apparaît dans les Propos rustiques (1547) de Noël Du Fail. Horry est aussi l'auteur de deux autres textes facétieux sur les crocheteurs (une altération courante de «clocheteur», sonneur des cloches) de Paris (1611 et 1612) (Fournier 1856, 235). Le Rabelais ressuscité a longtemps été considéré par les chercheurs comme une imitation ou un «pillage littéraire»de l'œuvre de Rabelais, comme un livre «alourdi, caricaturé, presque ridicule, [...] sans art et sans mesure»et comme une «fade parodie» (Côté 2016, 7; Oulmont 1908, 197; Sainean 1930, 22). Dans le même ordre d'idées, Neil Goodley affirme que «Horry [...] n'a aucune des subtilités stylistiques rabelaisiennes» et que le récit «témoigne d'[une] simplicité élémentaire et facile» (Goodley 1976, XXXIII). Marcel de Grève et Jean Céard affirment quant à lui que le Rabelais ressuscité «ne retient que l'élément bouffon et aventurier»du roman rabelaisien (De Grève et Céard 2009, 141). Toutes ces citations suggèrent que Horry tente donc de s'inscrire dans la lignée de Rabelais, mais qu'il lui manque le comique naturel, l'ingéniosité et la finesse de son modèle.

Néanmoins, grâce aux récentes études de Christine Arsenault sur les imitateurs ou «singes» de Rabelais, nous savons que cette perspective sur le Rabelais ressuscité est trop restrictive et unilatérale. Arsenault souligne en effet que «l'intérêt principal du R[abelais] R[essuscité] est la lecture en parallèle avec les écrits d'autres signes pararabelaisiens» (Arsenault 2016, 64). Selon elle, les imitateurs de Rabelais se lisaient les uns les autres, considérant l'ensemble narratif formé par les œuvres de Rabelais et celles de ses imitateurs comme un tout cohérent. C'est précisément pour cette raison que le Rabelais ressuscité est intéressant: le livre fait écho aux œuvres de Rabelais lui-même et à celles de ses contemporains, de ses imitateurs et d'autres tendances et genres littéraires de l'époque. Par exemple, le Rabelais ressuscité, en tant que parodie d'un roman de chevalerie, présente des rapports avec le Gargantua

\footnotetext{
Footnote 1 (continued)

tion comme «Horry 1611», sauf s'il s'agit d'une référence à l'introduction de Goodley. Nous nous référons à cette introduction comme «Goodley 1976».

2 Pour toutes les traductions ou explications des mots qui viennent du moyen français, nous utilisons les définitions et explications données par le Dictionnaire du Moyen Français (DMF) numérisé: CNRS et Université de Lorraine, Dictionnaire du Moyen Français, 2015, http://www.atilf.fr/dmf/.
} 
(1535) de Rabelais. Arsenault note également des allusions à la culture festive du $\mathrm{XVI}^{\mathrm{e}}$ siècle et de fortes influences entre genres ludiques qui ont en particulier affecté Nicolas de Horry, le Rabelais ressuscité étant par exemple influencé par la pronostication joyeuse (Arsenault 2015, 59-61).

Toutefois, nous voulons montrer ici que cette dernière catégorie d'influence ne se limite pas à la tradition des pronostications joyeuses. Nous défendons l'idée selon laquelle il existe au moins une autre tradition littéraire qui a beaucoup affecté la structure et le récit du Rabelais ressuscité: celle du mandement joyeux, qui est, selon Arsenault, un «genre littéraire parodique novateur dans la production pararabelaisienne» (Arsenault 2015, 146-154). Nous expliquerons tout d'abord les caractéristiques du mandement joyeux tel qu'il était pratiqué en France au $\mathrm{XV}^{\mathrm{e}}$ et au $\mathrm{XVI}^{\mathrm{e}}$ siècles. Ensuite, nous identifierons les mandements joyeux insérés dans le Rabelais ressuscité, et nous analyserons l'un d'eux en portant une attention particulière à son fonctionnement au sein du livre. Il s'agira plus spécifiquement de caractériser l'emploi de cet instrument parodique et d'expliquer son fonctionnement dans le récit.

\section{Les mandements joyeux en France: fictions juridiques parodiques}

La tradition des mandements joyeux en France est un sujet des études médiévales qui reste à explorer. Une étude générale sur les mandements joyeux manque encore, malgré les travaux de Jean-Claude Aubailly, et plus récemment de Jelle Koopmans et Paul Verhuyck, puis de Marie Bouhaik-Gironès et Katell Lavéant, qui ont contribué à signaler l'existence des mandements joyeux en analysant quelques exemples de ces textes parodiant des textes juridiques et des ordonnances royales (Aubailly 1976, 102-107; Verhuyck 1989; Koopmans 1997, 174-178; Bouhaïk-Gironès et Lavéant 2013). Dans cet article, nous esquissons donc avant tout une image globale de cette tradition littéraire, basée sur nos recherches actuelles.

Comme l'indique leur nom, les mandements joyeux sont des ordonnances joyeuses, c'est-à-dire ludiques, au contraire des mandements officiels du roi et de l'évêque. Comparé à une simple missive officielle, le mandement officiel est plutôt un acte par lequel une autorité s'adresse à ses sujets non seulement pour leur faire prendre connaissance d'une information, mais aussi pour leur donner un ou plusieurs ordres. En effet, le texte stipule qu'une action est attendue des destinataires, sous peine d'une punition. Les mandements joyeux font partie de la riche tradition des fictions juridiques parodiques (Bouhaïk-Gironès 2007, 153-188).

Le mandement joyeux est donc une imitation intentionnelle d'un mandement officiel qui «se conforme très strictement au code rhétorique de l'ordonnance officielle»(Verhuyck 1989, 89). À la fin du Moyen Âge, le mandement officiel compte douze éléments fixes, notamment les noms et titres de l'autorité qui le délivre, la description des destinataires, et l'ordre émis, le tout signé, daté et localisé: cette structure est le plus souvent scrupuleusement suivie dans les mandements joyeux. De manière générale, la parodie se caractérise par le fait que le public (lecteurs ou auditeurs) peut encore reconnaître le texte-modèle qui est imité. Cependant, le texte parodique a souvent pour but de communiquer une ou plusieurs idées qui 
n'entretiennent pas nécessairement de liens directs avec le texte-modèle. La parodie des mandements joyeux est ainsi de type exemplaire. Comme l'a montré John Yunck, ce type de parodie consiste en l'idée que le texte-modèle est l'arme qu' utilise et respecte le parodiste pour véhiculer certaines idées (Yunck 1963, 36-37). Les mandements joyeux évoquent et discutent entre autres des thèmes et personnages que nous pouvons lier à la culture festive et joyeuse de la fin du Moyen Âge (Verhuyck 1989, 99-100). Ainsi, le mandement joyeux s'inscrit dans une longue tradition de textes qui actualisent et concrétisent un mundus inversus (monde inversé ou renversé, Cocchiara 1981). Il s'agit par exemple du thème général de la fête, à l'éloge du vin, à l'oisiveté, au Carnaval et aux repues franches. De plus, la faim, la misère, la déchéance physique, la sexualité, l'immoralité et la satire des institutions officielles (Église, monarchie) jouent un rôle important dans le mandement joyeux.

Le mandement joyeux s'inscrit dans un registre de langue comique, qui vise principalement à provoquer le (sou)rire et l'amusement. Le mandement joyeux n'est pas véritablement un texte polémique, qui vise à diviser des groupes. Il n'emploie pas de ton violent, et ne présente pas d'attaque directe sur une personne ou sur un événement. Néanmoins, cela ne signifie pas qu'un mandement joyeux ne puisse pas transmettre un message critique dissimulé dans un format ludique.

\section{Identification des mandements joyeux dans le Rabelais ressuscité}

Précédemment, nous avons identifié les caractéristiques d'un mandement joyeux. Le mandement joyeux: (1) contient des ordonnances/appels joyeux, (2) connaît généralement une structure fixe de douze éléments, (3) est un texte parodique de type exemplaire, (4) contient des thèmes et des personnages (notamment une autorité) joyeux, (5) se caractérise par un registre de langue comique. D’après ces cinq éléments, nous décelons quatre mandements joyeux dans le Rabelais ressuscité: ${ }^{3}$

1. le mandement joyeux du chapitre XIIII, dans lequel Grangosier écrit une lettre à son père. Il lui envoie la preuve de son doctorat, un mandement joyeux donné par son professeur Monsieur Nemo. Nous désignerons dorénavant ce mandement par le titre «Copie des lettres de Docteur» car c'est dans le titre du chapitre XIIII que ce mandement joyeux est désigné par cette description (Horry 1611, 20-21).

2. le mandement joyeux du chapitre XIX. Il s'agit des «Ordonnances et Edicts», donnés par le roi Grangosier. Grangosier ordonne à ses sujets de célébrer chaque année la fête de Carnaval (Horry 1611, 36).

3. le premier mandement joyeux du chapitre XX. Il s'agit d'une lettre royale, écrite par Grangosier. Le mandement donne le privilège au sujet «Virecul Pallefesses» de

\footnotetext{
3 Selon le narrateur hétérodiégétique du Rabelais ressuscité, les quatre mandements joyeux constituent seulement une toute petite partie du nombre des mandements qui étaient distribués dans le royaume fictif de Grangosier. C'est notamment ce qu'il écrit dans le chapitre XIX: «la pluspart desquelles [ordonnances] ont esté perduës [...]» et «la plus grande partie d'iceluy [mandement] ne se peut lire pour estre tout rongé et mangé des souris» (Horry 1611,36).
} 
ramasser les ordures de Placevuide et de s'enrichir en les vendant (Horry 1611, 37-41).

4. le deuxième mandement du chapitre $X X$, qui porte sur les pratiques du charivari.

Pour illustrer la forme et les différents éléments d'un mandement joyeux tels que détaillés plus haut, nous proposons d'analyser la Copie des lettres de Docteur. Nous présentons ci-dessous le texte du mandement, avec l'identification des différents éléments formels entre parenthèses:

A tous aveugles qui ces presentes lettres verront. A tous sourds qui les entendront \& à tous muets qui d'icelles parler voudront [adresse]. Soit notoire \& manifeste, que pardevant nous tous les Docteurs de l'indocte faculté d'ignorance [invocation], assemblez en nostre auditoire ignorential sis à Paris en l'une des ruës de ladicte ville, ou pend pour enseigne l'image S. Nullus [suscription], Est comparu tres haut, tres-puissant \& tres impudent Prince, le Seigneur Grangosier, fils unique du Roy de Veautuerie, Duc de Nulleprovince, $\&$ gouverneur pour son ventre au chasteau de ses dents, lequel nous a dict \& remonstré que pour plusieurs considerations, \& principallement à cause que nostredite faculté d'ignorance, est la plus ancienne $\&$ plus estimee de toutes les autres, mesmement qu'il y a beaucoup plus grand nombre de personnes qui la suivent plustot que toutes autres scienes, qu'à cest effect il nous avoit fait assembler presentement, en nostredit auditoire, situé au lieu susdict, afin qu'il nous pleust l'interroger, \& examiner sur tous les plus indoctes points de nostre science, pour estre par nous receu Docteur en nostre communauté, apres qu'il nous aura faict apparoir son incapacité [préambule]. Pour à quoi parvenir nous a premierement exhibé ses lettres de maistres és asnes, en bonne et vallable forme, signees, insanus asinorum praefectus \& scellees in cera invisibili, ensemble un certificat de Monsieur Nemo, premier Philosophe loyal de l'université de Peudestudes, par lequel certificat appert de sa grande insuffisance, \& comme il a parachevé son cours en toute folie depuis long temps. Sur quoy \& apres qu'il nous a esté occultement apparu de ce que dict est, \& apres l'avoir par nous tres-ignoramment interrogé \& examiné, \& qu'il nous a tresindoctement respondu, sans faillir en un seul poinct sur les choses desquelles ne l'avons interrogé [narration]. Avons tous d'un accord dict \& disons que ledit seigneur Grangosier, attendu sa grande insuffisance \& incapacité, doit estre receu docteur en nostre faculté d'ignorance, \& lequel des maintenant y avons receu \& recevons, comme en estant capable. Et lui avons permis d'user $\&$ jouir de tous les privileges qui sont attribuez à ceux de nostredite societé, mesmes de porter les habillements tels qu'elle a accoustumé, sçavoir un bonnet carré verd de la hauteur seullement de dix pieds, pour esviter au scandale qui en pourroit advenir s'il le portoit plus hault, avec la soutane de satin jaune, duquel se vestent ordinairement les bergers, mesmes de porter sur iceux habillements du passement que les cordiers ont accoustumé faire pour des cordes de puits, \& de la mesme grosseur, pourveu qu'il n'en porte point davantage de soixante aulnes sur lesdicts habillements, sans qu'il en puisse estre repris en aucune façon par les muets: ny moqué par les aveugles [dispositif I], sur 
peine à iceux sçavoir ausdicts muets de ne plus parler, \& austicts aveugles de ne plus voir [pénitences I]. Et enjoint à tous escureurs de retraicts, pallefreniers, charcutiers, crocheteurs, vendeuses d'herbes, arracheurs de dents, fourbisseurs, savetiers, tripieres rotisseurs, crieurs de poudre pour faire mourir les rats \& les souris, vendeurs de grez, chaderonniers, maquignons \& revendeurs de chevaux bleuds, venderesses des harens en vie, crieurs d'allumettes, vinaigriers, vendeurs de moustarde, porteurs d'eau, vendeurs \& venderesses de noir à noircir, \& à tous autres de semblable qualité, qu'ils ayent à porter audict Seigneur Grangosier, tel respect comme ils feroient \& sont tenus faire à leurs propres serviteurs [dispositif II], sur peine d'encourir l'amende, en laquelle sont condamnez tous ceux qui mangent du pain sans en avoir, \& qui s'ennyvrent de boire de l'eau [pénitences II]. Et pareillement avons enjoinct à nostre greffier d'enregistrer les presentes au greffe de la reception des Docteurs de nostre communauté, pour y avoir recours, si quelqu'un doutoit de l'incapacité dudit seigneur Grangosier [corroboration]. En foi \& tesmoignage dequoy avons fait scelles les presentes de nostre grand sceau acoustumé, \& icelles fait signer par nostre greffier les an \& jour qu'elles furent faictes [datation], és presences de ignoble homme, Guillaume Plumepoire, maistre vendeur de noir à noircir, \& Thibaut Tournebroche, maistre vendeur d'allumettes demourans à Paris logez en l'hostel Dieu, \& plusieurs aumoins qui ont promis signer ces presentes dans mil ans prochainement \& esloignement venans s'ils sont en vie, signees indoctus greffier, \& scellees en cire rouge, ou jaune, ou blanche, ou verte [souscription].

Nous voyons que dix éléments sur douze des mandements officiels sont repris et que la salutation (avec la formule «Salut (et dilection»)) et la notification (avec la formule «faisons savoir») manquent. En effet, ce mandement contenant des privilèges passe immédiatement aux dispositifs.

L'autorité joyeuse prend une place importante dans le mandement joyeux. Ce personnage est celui qui donne les mandements ludiques et il est donc aussi important que le contenu pour distinguer le mandement joyeux du mandement officiel. Dans les mandements joyeux du Rabelais ressuscité, nous rencontrons deux autorités joyeuses: les professeurs de Grangosier (entre autres «Monsieur Nemo, premier Philosophe loyal de l'université de Peudestudes»(Horry 1611, 20)) et Grangosier lui-même. Les deux autorités joyeuses sont soutenues par leur arrière-ban (plusieurs professeurs dans le cas de Nemo, la famille, les greffiers, les lieutenants et notaires gourmands dans le cas de Grangosier). En plus de ces deux autorités joyeuses, nous retrouvons d'autres professeurs et étudiants de l'université, ainsi que les habitants du royaume de Placevuide de Grangosier. Ce ne sont pas nécessairement des personnages joyeux ou comiques. Le meilleur exemple de ce type de personnages est donné dans le chapitre X, dans lequel les camarades de classe de Grangosier s'avèrent être des étudiants très sérieux et motivés:

les compagnons [...] tousjours le picquoient et se mocquoient de luy, [...] disans que c'estoit une honte à luy [...] de ne rien sçavoir et que c'estoit un yvrongne insatiable [...], luy remonstroient qu'il falloit boire avec temperance, et d'ailleurs luy objectoient sa paresse [...] (Horry 1611, 13). 
Avec de telles contradictions entre les personnages et autorités joyeux et officiels, Nicolas de Horry joue sur les relations entre le monde officiel et le monde joyeux de son histoire. Ici, nous considérons ce «monde joyeux» comme un espace imaginaire et littéraire, présent dans et constitué par l'histoire du Rabelais ressuscité, comprenant tous les rites, valeurs, coutumes, images, préférences, etc. du personnage principal Grangosier. Le «monde officiel», en revanche, se présente dans le Rabelais ressuscité surtout en opposition au monde joyeux: sagesse, modération par rapport à l'alcool et à la nourriture, et travail acharné y sont les termes clés. C'est par exemple le monde principal des camarades de classe de Grangosier. À travers les interactions entre Grangosier et ses camarades de classe, Horry démontre que ces deux mondes, contradictoires à première vue, coexistent.

\section{La Copie des lettres de Docteur}

Des quatre mandement joyeux présents dans le Rabelais ressuscité, nous proposons d'analyser en détail la Copie des lettres de Docteur, le mandement concluant tous les chapitres sur l'éducation de Grangosier. Ce mandement est le seul qui soit parfaitement intégré dans l'histoire, c'est-à-dire qu'il y a un lien direct entre le contenu du mandement (la confirmation du doctorat de Grangosier et les privilèges qui en découlent) et celui des quatre chapitres précédents, portant sur l'éducation de Grangosier. À l'inverse, les trois autres mandements joyeux du Rabelais ressuscité sont plutôt des «cas isolés» : très peu d'informations sont données dans les chapitres pour signaler un lien thématique avec le contenu des mandements.

Dans le chapitre IX, nous lisons que Grangosier commence ses études à «l'université de Peudestudes», ainsi que son père Trousseviande l'a souhaité. Mais il se trouve que Grangosier n'étudie guère et ne veut que boire du vin. Lorsque le père de Grangosier apprend que son fils ne fait rien à l'Université de Peudestudes, il envoie Grangosier dans «une université florissante»à Paris. Là, Grangosier ne consacre pas plus de temps à ses études, mais il parvient à passer ses examens en trompant ses professeurs (c'est-à-dire en tenant sous le manteau «son livre ouvert où était écrite sa leçon»(Horry 1611, 17), chapitre XIII). Dans le chapitre XIV, Grangosier est toujours à Paris, où il termine son doctorat, lorsque son père lui envoie une lettre, dans laquelle il annonce la mort de sa mère, qui «s' estoit estranglée en mangeant une poire cuite fricassée au beurre verd» (Horry 1611, 19). Son père est désespéré. Grangoisier le console et répond de façon comique qu'il a terminé son doctorat: il ajoute à sa lettre la preuve du doctorat qu'il a reçue de ses professeurs, preuve qui est en réalité un mandement joyeux.

Bien que ce mandement joyeux semble à première vue être une simple confirmation de la réussite du doctorat de Grangosier, se rapprochant donc davantage de la lettre missive, les points distinctifs de ce mandement sont les privilèges joyeux donnés au géant par ses maîtres. Ces privilèges, ou droits particuliers, sont présentés sous forme de trois ordonnances ou dispositifs. Nous connaissons plusieurs autres 
exemples de privilèges joyeux dans la littérature française du XVI ${ }^{\mathrm{e}}$ et du XVII ${ }^{\mathrm{e}}$ siècle, octroyés sous forme d'un mandement joyeux. ${ }^{4}$

Dès le début de la Copie des lettres de Docteur, il est tout à fait clair qu'il s'agit d'un texte non-sérieux, dans lequel l'université en tant qu'institution est tournée en ridicule. Par exemple, la salutation et l'adresse «A tous aveugles qui ces presentes lettres verront. A tous sourds qui les entendront et à tous muets qui d'icelles parler voudront» (47-48) est, en soi, inutile: elle parodie la salutation régulière «À tous ceux qui ces présentes lettres verront, etc.». Du point de vue de la vie universitaire, il est possible que cette salutation se moque spécifiquement des étudiants juristes, qui, pendant leurs études de droit, apprenaient à écrire de telles formulations et aussi à les parodier (Bouhaik-Gironès 2007, 156-158). Les études universitaires sont ici ridiculisées par l'annonce que Grangosier est devenu docteur dans «nostre faculté d'ignorance» (56) de «l'université de Peudestudes»(67). L'étudiant Grangosier et ses professeurs sont eux aussi sévèrement moqués: Grangosier a été «tres-ignoramment interrogé et examiné, et il [...] a tres-indoctement respondu»(70-72), ce qui fait référence à sa soutenance de thèse, et l'on parle de «sa grande insuffisance et incapacité» (74) concernant la matière de son projet de thèse. Ces éléments sanctionnent donc de manière inversée le déroulement des études de Grangosier, en en louant le peu de mérites. Les privilèges donnés à Grangosier après avoir obtenu son doctorat se traduisent en mandements à ceux qui entendent ou lisent le document.

Dans ce texte, trois privilèges sont accordés à Grangosier, avec les trois ordonnances qui en découlent: le premier privilège autorise Grangosier à «porter les habillements» de la société des docteurs de la faculté d'ignorance. Ces vêtements sont «un bonnet carré verd de la hauteur [...] de dix pieds» (79-80) et une «soutane de satin jaune» (81). Au Moyen Âge le jaune et le vert symbolisaient le désordre, le dérèglement et, à la fin du Moyen Âge, la folie (Pastoureau 1986, 40). Dans la perspective du doctorat de Grangosier, cela signifie que ce dernier a donc le droit et le privilège de se déguiser en fou ou en bouffon. Le deuxième privilège concerne le respect que mérite Grangosier pour avoir fini ses études. Une liste de représentants de métiers divers est obligée de respecter le docteur Grangosier: «escureurs de retraicts, pallefreniers, charcutiers, crocheteurs, vendeuses d'herbes, arracheurs de dents, fourbisseurs, savetiers, tripieres, rotisseurs, crieurs de poudre pour faire mourir les rates et les souris, vendeurs de grez, chaderonniers, maquignons et revendeurs de chevaux bleuds, venderesses des harens en vie, crieurs d'allumettes, vinaigriers, vendeurs de moustarde, porteurs d'eau, vendeurs et venderesses de noir à noircir, et à tous autres de semblable qualité» (Horry 1611, 88-95). ${ }^{5}$ Remarquons que les groupes décrits ici

\footnotetext{
${ }^{4}$ Quelques exemples sont la Lettre d'escorniflerie, qui donne aux porteurs de ces mandements le privilège de beaucoup boire (Heist 1968), et les Lettres nouvelles contenantes le privilege et auctorite d'avoir deux femmes, qui, comme leur nom l'indique, donnent aux hommes le privilège d'être polygame (Rus 1995, 215-224).

5 «retraicts»: logis; «crocheteurs»: sonneur de cloches; «fourbisseurs»: celui qui confectionne, monte et polit les armes blanches et les armures; «grez»: grès; «bleuds»: la couleur bleue est un symbole de loyauté, de fidélité et de constance. Nous ne savons pas exactement pourquoi cet adjectif a été ajouté au substantif «chevaux»; «harens en vie»: le fait que des harengs vivants soient vendu est surprenant: habituellement, on vend des harengs morts, souvent fumés.
} 
sont toujours des petits métiers, qui rapportent peu d'argent. De plus, bon nombre de ces professions sont en lien avec la nourriture, notamment avec la viande, ce qui fait écho à la gourmandise de Grangosier. D'après le troisième privilège, les docteurs de la communauté académique de la faculté d'ignorance sont également obligés de respecter Grangosier en tant que docteur. Soulignons que cette communauté est une communauté académique parodique, soit l'inverse d'une communauté respectable. Leur patron est par exemple «S[anctus] Nullus» (52, Saint Néant, un saint imaginaire qui parodie (les vies) des saints et leur statut dans l'Église au Moyen Âge) et tous les docteurs y sont «indocte[s]» (ignorants, 49) (Merceron 2002, 787).

\section{Fonctionnement de la parodie à la Renaissance}

Dans son livre Excentricité et Humanisme: Parodie, dérision et détournement des codes à la Renaissance (2002), Patricia Eichel-Lojkine offre des clés qui permettent de proposer une interprétation plus précise de la Copie des lettres de Docteur. L'auteur analyse en effet les différentes fonctions de la parodie dans des textes de la Renaissance (Eichel-Lojkine 2002, 242). Selon elle, «il convient [...] d'interroger de manière critique l'opposition entre le sérieux et le comique, qui est empruntée à d'autres périodes et projetée sur la Renaissance [...]» (Eichel-Lojkine 2002, 20). Un point essentiel de cette approche - que nous adoptons également dans notre analyse - est l'affirmation de la coexistence d'un monde joyeux et d'un monde officiel dans la littérature de la Renaissance. Nous souscrivons également à l'observation d'Eichel-Lojkine selon laquelle la parodie est un instrument médiateur par excellence, qui permet à l'auteur de faire interagir le monde officiel et le monde joyeux. C'est notamment le cas dans l'œuvre de Rabelais:

[1]a fonction de la parodie dans l'écriture de Rabelais semble être de servir de médiation entre deux cultures incompatibles, deux modes de pensée radicalement différents [...]. L'étude de la parodie est peut-être la voie qui s'offre pour sortir du débat infécond entre tenants de l'humanisme et tenants du carnavalesque [...]; la novation radicale de Rabelais ne réside [pas] dans l'illustration ni de l'une ni de l'autre culture, mais dans l'usage de la parodie comme moyen d'articulation entre ces deux cultures [...] (Eichel-Lojkine 2002, 252).

La Copie des lettres de Docteur et les autres mandements joyeux du Rabelais ressuscité illustrent bien cette idée. D'une part, le mandement joyeux de la Copie des lettres de Docteur reprend à plusieurs niveaux certains thèmes de la culture festive ou, pour reprendre la terminologie de Bakhtine, de la culture carnavalesque. À un niveau très concret, on pense par exemple aux beuveries de Grangosier et de son professeur Nemo, ainsi qu'aux festins extraordinaires avalés par Grangosier. À un niveau plus abstrait, la Copie tire de la culture carnavalesque le topos du monde renversé: le monde universitaire est ici complètement illogique et contraire aux attentes sérieuses sur cette institution. Ajoutons aussi plusieurs références à la sottise, comme le fait que Grangosier devienne «maistre és asnes»(64-65) et que son privilège soit de pouvoir porter le costume d'un fou. D'autre part, le mandement 
joyeux reprend des aspects humanistes en introduisant par exemple des auteurs classiques (Caton, Épicure). L'accent mis sur le milieu universitaire vient également de cet esprit humaniste, dans lequel l'université prend une place prépondérante.

Par ailleurs, Eichel-Lojkine souligne que la thématique de l'opposition entre l'officiel et le joyeux issue du Moyen-Âge fonctionne différemment à l'époque prémoderne:

Force est de remettre en question l'opposition commode entre le discours édifiant et l'écriture de la dérision, pour arriver à rendre compte d'un fonctionnement largement inspiré des modes de pensée médiévaux, mais remodelé à la Renaissance [...] (Eichel-Lojkine 2002, 20).

Cette transformation est bien visible dans la Copie des lettres de Docteur. Ce mandement est explicitement situé dans un environnement universitaire, tandis que tous les autres exemples retrouvés de mandements joyeux du type des privilèges n'entretiennent aucun lien avec le milieu universitaire. En effet, tandis que les privilèges s'inscrivent dans la culture festive de la fin du Moyen Âge grâce aux thèmes du Carnaval et du mari battu, la Copie des lettres de Docteur replace le milieu universitaire dans le monde joyeux, en le présentant comme un lieu où le monde officiel et le monde joyeux interagissent continuellement. La figure de «Monsieur Nemo, premier Philosophe loyal de l'université de Peudestudes»(66-67) illustre bien ce processus de transformation. En effet, Horry reprend dans son texte un personnage et une thématique qui datent du XIII ${ }^{\mathrm{e}}$ siècle: Nemo (latin pour «personne, nul, rien, sans valeur») y est un personnage parodique qui apparaît souvent comme «la personnification de la folie [...] du monde» (Miernowski 1997, 117). Dans la traduction latine de la Bible, le mot «nemo» permettait à l'époque de nombreuses expressions comiques, comme «Nemo Deum vidit» (Personne ne voit Dieu) ou «Omnia Nemo potest»(Personne ne peut tout faire). Ce que la Bible dit de la faiblesse de l'homme est détourné pour faire la gloire de ce personnage imaginaire (Koopmans et Verhuyck 1987). Au cours du temps, le «Seigneur Nemo» ou «Monsieur Nemo» (66) est devenu un personnage récurrent dans les textes parodiant la Bible, comme dans le célèbre sermon joyeux Les grans et merveilleux faitz du seigneur Nemo (attribué à Jean d'Abondance, début du XVI ${ }^{\mathrm{e}}$ siècle). Dans le théâtre des $\mathrm{XV}^{\mathrm{e}}$ et $\mathrm{XVI}^{\mathrm{e}}$ siècles, Nemo «devient l'excuse des mauvais serviteurs, le responsable du désordre» (Koopmans 1997,81 ), ce qui a pour objectif de réaffirmer les vérités morales essentielles à la communauté des spectateurs (Miernowski 1997, 117). Dans la Copie des lettres de Docteur, la figure de Nemo est introduite et remodelée pour d'autres raisons encore. Nemo est tiré d'un contexte biblique puis positionné dans un milieu universitaire pour illustrer l'incapacité des professeurs de l'université de Paris, comme l'a également fait Rabelais dans Pantagruel et Gargantua. Monsieur Nemo devient, à travers les différents chapitres, le stéréotype du professeur ignorant et incapable, qui ne sait pas donner cours, ni diriger des thèses, et qui boit toujours trop. S'agit-il ici d'une attaque directe d'un groupe bien spécifique? Pour quelles raisons Horry utilise-t-il la parodie universitaire dans son roman?

Pour répondre à ces questions, nous reprenons de nouveau un cadre offert par Eichel-Lojkine, qui distingue différentes fonctions de parodies dans des textes de la Renaissance (entre autres le comique parodique, la parodie du code épique et 
la parodie subversive). Dans le chapitre IV de son étude, elle démontre comment certains passages de l'œuvre de Rabelais sont aussi bien parodiques que subversifs. Pour les passages concernant l'éducation (notamment l'éducation de Pantagruel à Paris dans Pantagruel, chapitre VII), elle constate qu'ils ne sont pas simplement «l'expression d'un credo humanisme» (Eichel-Lojkine 2002, 237), mais qu'ils sont également subversifs, au sens où ils relativisent et critiquent les pratiques et le discours des autorités universitaires à Paris. Elle suppose aussi que

[...] la subversion la plus vive, à la Renaissance comme peut-être encore aujourd'hui, ne se manifeste pas sous la forme d'une attaque frontale, mais s'opère au moyen de déplacements subtils, ambigus, qui laissent toujours ouverte l'interprétation (comme les différents tenants de la critique rabelaisienne le montrent bien) (Eichel-Lojkine 2002, 21).

Nous observons exactement ce modus operandi dans la Copie des lettres de Docteur. La critique n'est pas directe, elle est dissimulée dans le mandement joyeux ou parodique, qui n'est cependant pas un instrument univoque pour attaquer une institution ou une personne. Le sens du mandement joyeux est, à des degrés divers, équivoque et ouvert à plusieurs interprétations. Néanmoins, une interprétation subversive du mandement joyeux semble tout à fait probable: avec l'avènement de l'humanisme au $\mathrm{XV}^{\mathrm{e}}$ et au $\mathrm{XVI}^{\mathrm{e}}$ siècles, l'enseignement scolastique était de plus en plus remis en question, mais l'université de Paris restait tout de même imperméable aux idées nouvelles. Il nous semble que la Copie des lettres de Docteur doive être étudiée à la lumière de ce débat humaniste, effectué notamment au temps de Rabelais (EichelLojkine 2002, 237-242).

Dans les chapitres et le mandement concernant l'éducation de Grangosier, Horry reproche dans le même style que Rabelais l'enseignement scolastique, et critique l'enseignement purement livresque («sçavoir est de ne point ouvrir ses livres que bien rarement»(Horry 1611, 12). À travers le professeur Nemo, Horry s'adresse tout d'abord aux professeurs, mais il critique également le système d'enseignement médiéval (les arts libéraux), en particulier le trivium. Les étudiants comme Grangosier apprennent notamment les mécanismes de la langue (grammaire), la faculté d'argumenter (dialectique, logique) et comment communiquer (rhétorique). Dans la Copie des lettres de Docteur, Horry rejette les trois arts du trivium. Le fait que Grangosier «ne pouvoit encores decliner musa, ni conguguer amo» (Horry 1611, 13) est une indication que la grammaire en tant que discipline est remise en question. La dialectique et la logique sont aussi ridiculisées dans le Rabelais ressuscité, sous forme de leçons pour les étudiants, qui portent sur les commentaires des textes classiques: Grangosier ridiculise la logique des commentaires en expliquant (et pratiquant) très littéralement l'expression de Caton Bibe quod possis (bois autant que tu pourras) (Horry 1611,13) et le chapitre De modo bibendi (sur les façons de boire) d'Épicure. Finalement, la rhétorique en tant que discipline est critiquée à travers les conversations entre Grangosier et les autres étudiants (les deux partis ne se comprennent jamais), ainsi qu'à travers la structure du mandement joyeux, qui se moque de la rhétorique du mandement officiel. Les discussions inutiles entre Grangosier et ses professeurs (qui sont «tres-ignorra[nts]»(71)) s'inscrivent aussi dans cette critique de la rhétorique. 
Une question importante reste à élucider: pourquoi une telle critique du milieu universitaire (qui se rapproche de celle de Rabelais) est-elle encore proposée à l'époque de la publication du Rabelais ressuscité, c'est-à-dire presque un siècle après Rabelais? L'enseignement universitaire s'était déjà largement détourné de la scolastique médiévale et s'était adapté aux idées de l'humanisme en matière d'éducation.

Afin de donner une réponse à cette question, il nous faut revenir aux études de Christine Arsenault. Arsenault a démontré que les œuvres de Rabelais et celles de ses imitateurs forment un ensemble narratif cohérent et complémentaire. Cela implique que les histoires écrites après les œuvres de Rabelais lui-même s'inscrivent dans le même univers, un univers qui se caractérise par une interaction permanente entre le monde officiel et le monde joyeux. Les histoires rabelaisiennes présentent les mêmes thématiques et styles d'écriture, et respectent la chronologie de tous les événements ayant eu lieu dans cet univers. Dans cet ensemble, le Rabelais ressuscité présente l'histoire d'un ancêtre de Pantagruel, ce qui implique que l'enseignement universitaire était au temps de Grangosier au moins aussi scolastique qu'au temps de Pantagruel. Horry a donc dû adopter le cadre universitaire et la critique subversive tels qu'esquissés par Rabelais dans son Pantagruel pour garder la cohérence de l'univers rabelaisien. Il ne s'agit donc pas d'une attaque directe des professeurs parisiens du XVII ${ }^{\mathrm{e}}$ siècle: l'intensité du propos du mandement joyeux ou parodique reste principalement ludique.

Néanmoins, dans ce cas, il faut dépasser l'explication d'Arsenault. Horry intensifie délibérément la critique du milieu universitaire en déployant l'instrument parodique du mandement joyeux à la fin de plusieurs chapitres consacrés à l'éducation de Grangosier. Une approche institutionnelle du Rabelais ressuscité permettrait de trouver les autres motivations possibles de Horry. Dans cette perspective, la question centrale concerne le(s) public(s) cible(s) ou le(s) destinataire(s) de ce texte. Tout d'abord, il est possible que Horry ait écrit ce texte pour un public cultivé qui non seulement connaissait bien les textes de Rabelais, mais qui était également familier de la critique de Rabelais du système universitaire. Ces lecteurs ont probablement étudié à la Sorbonne. Les références à l'enseignement scolastique de cette université font ainsi appel à la mémoire collective des lecteurs, même s'ils ont suivi une formation plus humaniste que scolastique. Les chapitres du Rabelais ressuscité consacrés à l'éducation se lisent comme des anecdotes ludiques se référant aux représentations du passé connues de ce public savant.

Un deuxième groupe de lecteurs possible est un public populaire qui a désormais accès aux textes de Rabelais grâce à leur dissémination à des couches plus larges de la société. L'étude centrale de Marcel de Grève et Jean Céard a montré que Rabelais fascinait des lecteurs de plus en plus nombreux (De Grève et Céard 2009). Ce public est supposé connaître le point de vue de Rabelais sur l'enseignement scolastique. Dans ce cas on peut parler d'une popularisation des textes et des idées de Rabelais dans des éditions de plus en plus bon marché au début du XVII ${ }^{\mathrm{e}}$ siècle.

Nous sommes encline à penser que Horry a écrit le Rabelais ressuscité pour un public cultivé, même si les deux publics mentionnés ne s'excluent pas nécessairement l'un l'autre. La raison principale qui justifie cette hypothèse est que trois autres histoires pararabelaisiennes datant de la même période (1610-1615) 
s'inscrivent certes dans l'univers comique de Rabelais, mais se réfèrent en même temps aux débats et conflits contemporains comme l'assassinat d'Henri IV en mai 1610, l'accession au trône de Louis XIII en 1611 et la Réforme. Ces histoires pararabelaisiennes étaient notamment destinées à un public cultivé et engagé, qui s'intéressait à ces débats politiques et religieux. Les œuvres qui ne sont pas pamphlétaires, comme les textes destinés au grand public, montrent la dissimulation ou l'encodage d'un message politique, discernable par un lecteur bien averti (Lestringant 2005, 122). ${ }^{6}$ Il semble que le même phénomène s'applique au Rabelais ressuscité. On peut donc considérer le Rabelais ressuscité comme un récit engagé, voire comme un roman ou livre à clé, dont il existe plusieurs clés possibles. Ce livre à clé se réfère à une fiction dans laquelle se cachent des personnes réelles que le lecteur peut reconnaître. En revanche, les mandements joyeux ne se présentent pas nécessairement comme des énigmes à résoudre, mais plutôt comme des textes purement amusants. Les nombreuses métaphores comiques et érotiques dans les mandements joyeux en sont un exemple. ${ }^{7}$ La signification des mandements joyeux dans le livre n'est donc pas évidente au premier abord. Elle dépend vraisemblablement des débats contemporains dans lesquels Horry a voulu s'inscrire, ainsi que de l'esprit comique de Rabelais que Horry a souhaité faire résonner.

Cela nous amène également à une autre possibilité: les mandements joyeux dans le Rabelais ressuscité ont pu avoir circulé de manière autonome avant d'être inclus dans le récit du Rabelais ressuscité. Cette hypothèse expliquerait les rapports finalement très ténus entre le récit et les mandements joyeux. Il nous semble possible que les mandements joyeux traitent des mêmes questions que le Rabelais ressuscité et les autres récits pararabelaisiens (le Carnaval, le charivari et l'université), mais que les correspondances s'arrêtent là. Dans cette optique, l'ajout des mandements joyeux au récit du Rabelais ressuscité peut être considéré comme une stratégie narrative de Horry pour rendre son texte plus attrayant.

\section{Conclusion: pourquoi le mandement joyeux?}

Dans le Rabelais ressuscité, Nicolas de Horry a pris la liberté de changer la façon dont le milieu universitaire est tourné en ridicule en déployant l'instrument parodique du mandement joyeux. À notre avis, la raison principale pour laquelle Horry a choisi d'utiliser le mandement joyeux est pour y résumer sa critique sur le système universitaire dans le but de l'intensifier. Le mandement est l'apogée de la parodie du milieu universitaire et il conclut de façon ludique les cinq chapitres sur l'éducation universitaire. Tous les aspects de critique déjà stipulés dans les chapitres

\footnotetext{
${ }^{6}$ Christine Arsenault a retrouvé quatre imitations de Rabelais parues entre 1610 et 1615 . Elle les a analysées du point de vue de la Réforme dans sa présentation «Panurge contre les Huguenots: Guillaume Reboul et sa navigation pararabelaisienne catholique» à la conférence de la RSA à Chicago en mars 2017. 7 Par exemple, dans le texte de Horry, la porte ouverte est une métaphore de la femme ouverte à plusieurs rapports sexuels; l' «Ambassadeur d'amour»est l'image du mari battu qui facilite plusieurs relations; les chaussures et les manteaux que doit ranger le mari battu sont la métaphore d'un triangle amoureux.
} 
précédents (chapitres IX à XIV, qui évoquent la stupidité et l'ignorance des professeurs, l'aversion de Horry pour les trois arts du trivium, les possibilités de tromper les professeurs) sont rassemblés dans le mandement joyeux. Néanmoins, tandis qu'il y a dans l'œuvre de Rabelais des références au milieu universitaire contemporain à travers les histoires fantastiques de Pantagruel, ces références n'existent plus de manière aussi claire au temps de Horry, puisque ce dernier écrit presque cent ans après Rabelais. Il ne s'agit donc plus d'une critique directe mais plutôt d'un appel à la mémoire collective du public cible, c'est-à-dire un public cultivé qui se souvient ou a entendu parler de l'enseignement pré-humaniste. De plus, il est possible que les mandements joyeux, dont la Copie des lettres de docteur, mais aussi les autres exemples dans le Rabelais ressuscité, aient d'abord circulé de façon autonome avant d'être insérés dans l'histoire du Rabelais ressuscité. Il s'agit finalement des mandements joyeux sur des thèmes qu'on rencontre souvent dans des textes parodiques, comme la figure de Nemo, le Carnaval et le mari battu. Il est fort probable que Horry ait ajouté les mandements pour les mettre au service de son récit et pour le rendre ainsi plus attrayant.

Funding The funding for this research was provided by NWO, the Netherlands Organisation for Scientific Research.

Open Access This article is distributed under the terms of the Creative Commons Attribution 4.0 International License (http://creativecommons.org/licenses/by/4.0/), which permits unrestricted use, distribution, and reproduction in any medium, provided you give appropriate credit to the original author(s) and the source, provide a link to the Creative Commons license, and indicate if changes were made.

\section{Références}

Arsenault, C. (2015). Les "Singes de Rabelais": transfictionnalité et postérité littéraire de l'œuvre rabelaisienne (1523-1619). Thèse de doctorat: Université Paris-Sorbonne et Université du Québec à Rimouski.

Arsenault, C. (2016). Réécrire Grangosier : le cas du Rabelais ressuscité de Nicolas de Horry. @nalyses. Revue de critique et de théorie littéraire, 11 (2), 50-67.

Aubailly, J.-C. (1976). Le monologue, le dialogue, et la sottie. Paris: Honoré Champion.

Bouhaik-Gironès, M. (2007). Les clercs de la Basoche et le théâtre comique: Paris, 1420-1550. Paris: Honoré Champion.

Bouhaiik-Gironès, M., \& Lavéant, K. (2013). Le Mandement de froidure de Jean Molinet: la culture joyeuse, un pont entre la cour de Bourgogne et les milieux urbains. In J. Devaux, E. Doudet, \& E. Lecuppre-Desjardin (Eds.), Jean Molinet et son temps (pp. 67-82). Brepols: Turnhout.

Cocchiara, G. (1981 [1963]). Il mondo allá rovescia. Turin: Boringhieri.

Côté, M. (2016). L'écriture hypertextuelle à la Renaissance. Des Chroniques gargantuines au Rabelais ressuscité de Nicolas Horry. Revue Lä̈us, 9, 7-20.

de Grève, M., \& Céard, J. (2009). La réception de Rabelais en Europe du XVI $I^{e}$ au XVIII siècle. Paris: Honoré Champion.

de Horry, N. [Thibaut le Nattier] (1867), Rabelais Ressuscité, Récitant Les Faicts et Comportements Admirables Du Très-Valeureux Grandgosier, Roy de Place-Vuide. Traduict de Grec Affricain En François Par Thibaut Le Nattier, G. Brunet (Ed.). Genève: Gay et fils.

de Horry, N. [Thibaut le Nattier] (1976). Rabelais Ressuscité, N. Goodley (Ed.). Exeter: University of Exeter. 
Eichel-Lojkine, P. (2002). Excentricité et humanisme: parodie, dérision et détournement des codes à la Renaissance. Genève: Librairie Droz.

Fournier, E. (1856). Variétés historiques et littéraires: recueil de pièces volantes rares et curieuses en prose et en vers, Tome IV. Paris: P. Jannet.

Heist, W. (1968). Sermon joyeux and polemic: Two sixteenth-century applications of the legend of the fifteen signs. Carolina: University of North Carolina Press.

Koopmans, J. (1997). Le théâtre des exclus au Moyen Age. Paris: Imago.

Koopmans, J., \& Verhuyck, P. (1987). Sermon joyeux et truanderie (Villon, Nemo, Ulespiègle). Amsterdam: Rodopi.

Lestringant, F. (2005). Une liberte feroce : Guillaume Reboul et Le Nouveau Panurge. In I. Moreau \& G. Holtz (Eds.), «Parler librement». La liberté de parole au tournant du XVI et du XVII siècle. Lyon: ENS Editions.

Merceron, J. (2002). Dictionnaire des saints imaginaires et facétieux. Paris: Seuil.

Miernowski, J. (1997). Signes dissimilaires: la quête des noms divins dans la poésie française de la Renaissance. Genève: Droz.

Oulmont, C. (1908). Le Rabelais ressuscité (1611). Revue des études rabelaisiennes, 1908, 196-200.

Pastoureau, M. (1986). Figures et couleurs: études sur la symbolique et la sensibilité médiévales. Paris: Léopard d'or.

Rus, M. (1995). De la conception à l'au-delà: textes et documents français d'un siècle qui n'en est pas un (1450-1550). Amsterdam: Rodopi.

Sainean, L. (1930). L'influence et la réputation de Rabelais: interprètes, lecteurs et imitateurs, un rabelaisien. Paris: J. Gamber.

Verhuyck, P. (1989). Les mandements joyeux et le Mandement de Bacchus, Anvers, 1580. In M. Lazard (Ed.), Aspects du théâtre populaire en Europe au XVI siècle (pp. 89-103). Paris: Sedes.

Yunck, J. A. (1963). The two faces of parody. Iowa English Yearbook. Literature in High School, 8, 36-37.

Publisher's Note Springer Nature remains neutral with regard to jurisdictional claims in published maps and institutional affiliations. 